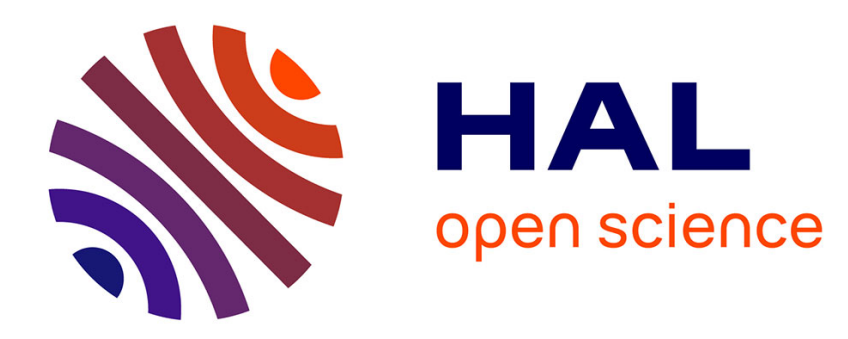

\title{
Cérémonies autour du thème de la navigation à Amathonte
}

\author{
Aurélie Carbillet
}

\section{To cite this version:}

Aurélie Carbillet. Cérémonies autour du thème de la navigation à Amathonte. Cahiers du Centre d'Etudes Chypriotes, 2005, 35, pp.77-88. hal-00121356

\section{HAL Id: hal-00121356 https://hal.science/hal-00121356}

Submitted on 20 Dec 2006

HAL is a multi-disciplinary open access archive for the deposit and dissemination of scientific research documents, whether they are published or not. The documents may come from teaching and research institutions in France or abroad, or from public or private research centers.
L'archive ouverte pluridisciplinaire HAL, est destinée au dépôt et à la diffusion de documents scientifiques de niveau recherche, publiés ou non, émanant des établissements d'enseignement et de recherche français ou étrangers, des laboratoires publics ou privés. 
Article paru dans le Cahier du Centre d'Etudes Chypriotes, 2005, n 35 


\title{
CÉRÉMONIES AUTOUR DU THÈME DE LA NAVIGATION À AMATHONTE
}

\author{
Aurélie CARBILLET
}

\begin{abstract}
A terracota model hoat of the Archaic period, found in the sea off Amathus, was published in 1999 by L. Basch who gave a detailed commentary from the perspective of its naval architecture. The fact that the vessel was in this particular instance oceupied hy seven people justifies making an iconograhic study so as to determine the significance of this kind of offering which was particularly common at the site of Amathus. The probable presence of a king-like figure amongst the persons on board allows new hypotheses to be put forward on the cults connected with sailing and the role played by the ruler.
\end{abstract}

Cet article porte sur un modèle de bateau en terre cuite, publié par L. Basch en 1999, et dont l'interprétation iconographique mérite, à mon sens, quelques approfondissements : il s'agit du «modèle Kakoulli » de la Fondation Piéridès (Fig. 1-5) ${ }^{1}$. Cet objet a été retrouvé en mer, au large d'Amathonte. L. Basch le date du ChyproArchaïque II d'après des critères morphologiques pertinents. Son étude va nous permettre d'aborder la question de la possible existence, à l'époque archaïque et dans le royaume d'Amathonte, de fêtes ou de rites liés à la navigation des bateaux, el aux voyages effectués sur une mer parfois capricicuse.

\section{Les bateaux chypriotes en terre cuite: une production spécifique}

Parmi les sujets représentés par les coroplastes chypriotes, les bateaux occupent une place non négligeable ${ }^{2}$. Quand ils ne sont pas issus des pillages intempestifs qu'a connus l'île auX XVIIIC et, surtout, XIXe siècles, ces objets ont été mis au jour sur plusieurs sites (Amathonte, Salamine, Kition, Maroni, Kazaphani, etc.), fréquemment en contexte funéraire, mais aussi dans certaines zones urbaines (sanctuaires, palais, remparts, ete.). Leur production semble commeneer au Bronze Moyen (date des plus 
anciens exemplaires retrouvés à ee jour) "et décliner au Chypro-Classique I. Elle s'étend done sur une durée asséz longue, d'environ 1500 ans $(c a$. 190()-400) av. J.-C.). Tous les types de navires sont représentés : petits et grands navires de guerre, navires de commerce de types divers, ou simples barques de pêche ${ }^{4}$. Amathonte se singularise par rapport aux autres villes, en raison du nombre important de modèles découverts (cnviron une quarantaine) et de leur diversité morphologique.

\section{La production amathousienne}

Deux zones du site ont livré des bateaux en terre cuite : l'acropole et les nécropoles. Les modèles découverts sur l'acropole d'Amathonte sont tous dans un état très fragmentaire, au point que leur identification en tant que tels est parfois incertaine. Ils sont tous de facture assez simple, en forme de barques, avec parfois quelques aménagements indiqués (gouvernail, rame). Trois modèles de hateaux ont ainsi été découverts sur le chantier du palais ${ }^{5}$. Ils datent du début de l'époque classique. Ce sont des barques, sans personnage représenté à l'intérieur, mais quelques aménagements sont parlois indiqués (plate-forme, barre transversale, etc.).

Trois autres modèles faisaient également partie du mobilier de la Terrasse Ouest ${ }^{6}$. Ils sont datés de l'époque archaïque. Ce sont toujours des barques sans aménagements intérieurs. Un seul modèle comporte un personnage, adossé à l'une des extrémités du bateau.

Sept modèles de bateaux ont été découverts dans différentes zones du sanctuaire d'Aphrodite ${ }^{7}$, et deux autres ont été mis au jour parmi le matériel qui comblait l'entrée d'une petite grotte naturelle, située dans la partic sud-est du sanctuaire, contre le mur du temenos paléochrétien ${ }^{8}$. Ils sont datés de l'époque archaïque. Ce sont des barques, dont la forme originelle est presque méconnaissable. Aucun personnage n'est représenté à l'intérieur de ces bateaux. Quelques aménagements intérieurs ou décorations externes sont parfois indiqués (quille, pastilles appliquées sur la coque, gouvernail, etc.). Un modèle, pourtant, diffère des autres: le fragment montre une barque plate, dont l'extrémité pointue est entourée d'un boudin d'argile imitant vraisemblablement un lien

3. Un exemplaire fragmentaire trouvé dans une tombe à Politiko-Lambertis (Westerberg 1983, p. 10, fig. 2) et un autre, conservé au Louvre (inv. AM 972), de provenance inconnue et aujourd'hui entièrement restauré (Göttlicher 1978, p. 34, fïg. 143).

4. Nous en avons dressé le catalogue dans un mémoire de Maîtrise (inédit).

5. Hermary $20(x)$, p. 53, nos $270-272$.

6. Ibid.. p. 54, no. $273-275$.

7. Ibid., p. 54, n"s $276-282$.

8. Ibid.. p. 54, nos $28.3-284$. 
en papyrus ${ }^{9}$. Sa forme est très similaire aux représentations des bateaux cérémoniels égyptiens ${ }^{10}$. Il est l'unique exemplaire de ce type retrouvé à Chypre à ce jour.

La majorité des modèles de bateaux en terre cuite provient des nécropoles amathousiennes 11 , trouvés principalement dans des tombes datées de l'époque archäque ${ }^{12}$. Les modèles mis au jour dans ces tombes sont de formes très diverses, mais on peut tout de même distinguer deux types : d'une part, les navires de guerre, de forme générale similaire ${ }^{13}$, avec la proue en forme de tête de bovidé et un grand ail noir surmonté d'un sourcil noir peint sur la coque; d'autre part, les barques avec une coque plus ou moins allongée et des extrémités relevées. Ces deux types se retrouvent dans des proportions à peu près semblables dans les nécropoles : dix navires de guerre y sont attestés et huit barques.

Le parallèle égyptien et le rapport avec la navigation céleste semblent apporter l'explication la plus satisfaisante à la présence de ces objets dans les nécropoles chypriotes ${ }^{14}$. En Égypte, c'est une très ancienne coutume que de munir le défunt de barques ou bateaux pour qu'il puisse naviguer jusqu'à l'au-delà ${ }^{15}$. Pour les pharaons ou les nantis, il s'agit de modèles de bateaux en bois, de taille réelle, et plus simplement de modèles en terre cuite, bois, pierre ou ivoire, pour le commun des mortels ${ }^{16}$.

\section{Ibid., p. 54, n² 280 , pl. 20 .}

10. Voir Jones 1990 , p. 5.

11. Tombes $\mathrm{n}^{\mathrm{os}} 3$ (BCH 101, 1977, p. 765-766); 73 (Murray et al. 1900, p. 112 et 119 , fig. 164,17 ) ; 83; 88 (ibid., p. 114, lig. 165, 6 et p. 120); 95 (ibid., p. 120); 176 (ibid., p. 113, fig. 164, 22); 130 (Karageorghis 1987, $\mathrm{n}^{\circ} 137$, pl. XXVII); 183 (Karageorghis 1996, p. 75, cat. $\mathrm{n}^{\circ} \mathrm{V}[\mathrm{b}] 20$, pl. XLIII) ; 210 (Karageorghis 1996, p. 74, fig. 59) ; 429 (BCH 110, 1986, p. 834, fig. 41); 441 (BCH 111, 1987, p. 658, fig. 57) ; 521 (BCH 111, 1987, p. 719, fig. 173); 602 (BCH 116, 1992, p. 799, fig. 17).

12. Seule une tombe, T. 521 , est datée de l'époque géométrique.

13. À l'exception des modèles des tombes 429 et 602 , qui présentent tous deux une paire de boucliers fixée sur les deux rebords.

14. Dans la religion égyptienne, les bateaux sont également les attributs d'Osiris, et ils sont déposés dans les tombes afin de représenter la navigation eschatologique des défunts et la présence de ce dieu. Osiris est le dieu de l'au-delà, le dieu garant de toute résurrection humaine. L'étude du matériel associé à ces objets laisse penser que les Amathousiens partageaient cette croyance. Osiris, en tout cas, est loin d'être inconnu dans le royaume. Outre

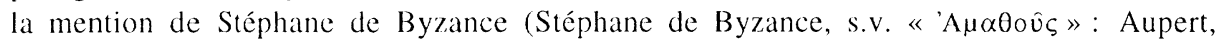
Hellmann 1984, p. 22, n 42a), une tête d'Osiris a été découverte parmi le mobilier funéraire d'une tombe (non identifiée, voir Murray et al. 1900, p. 112, fig. 164, n 8), ainsi qu'une figurine de Ptah-Socharis-Osiris dans la tombe 88 , laquelle a livré aussi un modèle de bateau (ibid., p. 113, fïg. 165, $\mathrm{n}^{\circ} 4$ ). Cette dernière contenait également une autre terre cuite qui représente «une figure à caractère égyptien» (ibid., p. 113, fig. 165, n 8). Les objets d'importation égyptienne ou à caractère égyptisant sont aussi présents en quantité assez importante dans les nécropoles, sous la forme de scarabées (même si la grande majorité est de fabrique locale chypriote), de figurines en terre cuite, etc.

15. Jones 1990, p. 1-2.

16. Voir des exemples dans Göttlicher 1978, pl. 6-8. 
À Amathonte, semble-1-il. les coroplastes ont privilégié la représentation du bateau en tant qu'objet, puisqu'il est rarement pourvu de personnages 17. Le "modèle Kakoulli » se distingue, quant à lui, en ce qu'il introduit une tout autre dimension : il renvoie à une scène historiée, ce qui n’apparâit pas sur les modeles amathousiens découverts sur l'acropole et dans les nécropoles. La représentation fait référence à une action, une image de la vie ou de la mort, un mythe, ete., et c'est la nature de cette scène dans son ensemble qu'il convient ici de déterminer.

De même, les deux modèles de bateaux chypriotes les plus anciens - datés du Bronze Moyen - associent déjà ces objets à une scène historiée. Le premier provient de Politiko-Lambertis ${ }^{18}$. Il est malheureusement dans un état très fragmentaire. Les fragments conservés montrent au moins ciny personnages: seul l'un d'entre cux est complet, des quatre autres il ne reste que la partie inféricure (bassin et jambes). Tous sont assis sur le rebord de l'embarcation, pieds à l'extérieur, les deux mains jointes pour le personnage entièrement conservé. Le second est un bateau de forme similaire, presque entièrement conservé, mais très restauré et de provenance inconnue ${ }^{19}$. Il est d'une facture assez exceptionnelle pour l'époque. Sa forme générale est celle d'un bol avec les extrémités en forme de cornes. L'équipage est composé de huit personnages, tous appuyés sur les bords de l'embarcation et vraisemblablement figurés comme ils naviguaient en réalité.

Il existe également d'autres modèles de bateaux, datés de l'époque archaïque, qui comprennent plusieurs personnages ${ }^{20}$ (jamais plus de trois), mais le «modèle Kakoulli », en raison de sa facture très soignée, de son souci dans le rendu des détails, et du nombre de personnages qu'il comporte, ne connaît pas d'équivalent contemporain.

\section{Le «modèle Kakoulli » dans le contexte amathousien}

Je ne reviendrai pas ici sur les caractéristiques morphologiques de ce modèle, très bien détaillées dans l'étude de L. Basch 21 . Il faut cependant signaler que ce bateau est, selon l'auteur, "le plus riche d'enseignements en matière d'architecture navale » 22 . En outre, la scène qui se déroule à bord lui donne une signification très particulière, à laquelle il convient maintenant de s'intéresser.

17. Par comparaison avec les modèles découverts à Salamine et à Kition qui, eux, comportent pratiquement toujours un personnage, parfois en position de "rameur ", parfois p. 129, cat. n'II(vi)1, 2 et 5 ; pour Kition, BCH 104, 1980,1984 et Karageorghis 1995,

18. Il date du Bronze Moyen. Voir Westerberg 1983, p. 10, fig. 2.

19. Voir Göllicher 1978, p. 34, fïg. 143. 20. Par exemple, Musée de Chypre inv. n"s 1937/VI-8/3, 1953/XII-30/6 et 1946/XII-23/1 ;
Muséc de Famagouste inv. n 1933/IX-13/2.

21. Basch 1999, p. 46-54.

22. Hid., p. 58. 
C'est un bateau de dimensions importantes (1. $45.5 \mathrm{~cm} ; 1.17 .5 \mathrm{~cm}$ ), qui témoigne d'un soin particulier apporté à la représentation et au rendu de nombreux détails. A bord sont lïgurés pas moins de sept personnages. Le modele possède une figure de proue qui ressemble à une protomé de grifton el qui pourrait être apotropä̈que 23 . même si ce motif est jusqu’ici inédit dans le répertoire des navires de la Méditerranée antique. La poupe se termine par une espèce de corne tournée vers l'intérieur, qui représente probablement une tête de volatile $2 t$.

La scène ligurée, d'emblée, attire l'attention. Sept personnages sont embarqués sur ce navire, regroupés aux deux extrémités, quatre à proximité de la proue el trois autres à l'arrière, près de la poupe. Notons aussi la présence d'un dernier personnage, à l'écart de ces deux groupes, qui donne une touche asse\% pittoresque à cet ensemble puisqu'il est juché sur une échelle à bâbord (Fig. 1).

Intéressons-nous d’abord au premier groupe de personnages, celui qui est placé a l'avant du bateau (fig. 4). A la proue, trois personnages aux bras étendus, dans une attitude très inconfortable - qui rend peu probable le fait qu'ils aient pu jouer un rôle quelconque dans les manceuves du navire -, entourent un quatrieme, debout sur le gaillard avant, nettement plus grand que les autres. Ce dernier se distingue eneore des trois autres par sa coifure: un turban. Dans une étude iconographicjue du sarcophage royal d'Amathonte, sur lequel est aussi figuré un personnage portant ce même type de turban, Th. Petit a démontré, à la suite de J.H. et S.H. Young, d'A. Hermary el de R. Senfi, que ectle coiffure n'était autre que la mitra que portaient les rois chypriotes à l'époque archä̈que ${ }^{25}$. Notre personnage serait done le roi, ce qui est tout à fait plausible vu la datation de ce modè à la pleine époque des royaumes. Mais le roi a une attitude, une fonction particulière sur ce bateau : il tient la rambarde de sa main gauche et fait ce qui semble être un geste d'adoration ou de bénédiction avec sa main droite, en direction de tout ce qui lui fait face, e’est-à-dire le navire lui-même el le second groupe de personnages juchés sur la dunette, à l'arrière du bateau. À sa gauche se dresse un thymiatérion, qui donne à la scène une évidente dimension cultuelle. L'attitude étrange des trois autres personnages qui sont derrière lui invite à se demander s'ils ne sont pas tout simplement des acolytes de ce roi/prêtre, qui essaient de masquer aux yeux des profanes la prière ou la cérémonie de bénédiction qui se déroule derrière eux.

Le second groupe, liguré sur la dunette, à l'arrière du bateau, est tout aussi étonnant et intéressant (Fig. 5). Il s'agit de trois personnages côte à côte. Tous ont les cheveux longs, mais le personnage eentral, plus grand que les autres, porte lui aussi la mitra 26. Il peut donc également s’agir de la représentation du roi, mais dans une attitude

23. Ihid., p. 46-47.

24. Ibid., p. 47.

25. Petit 1996, p. $111-113$ et Petit 2003, p. 6-7.

26. I. Basch avait bien repéré le turban que porte ce personnage, concluant qu'il devait simplement s'agir du "capitaine" du navire (Basch 1999, p. 57). 
complètement différente de son homologue de l'avant 27 . Il regarde au-dehors du navire, en direction de la mer ou du large, et ne fait aucun signe d'adoration.

Ce modele, exceptionnel tant par sa représentation que par le contexte de sa découverte - au large des côtes amathousiennes -, suggère l'existence de rituels au cours desquels le roipprêtre d'Amathonte invoquait une divinité euploia (Aphrodite Euploia?) afin d'assurer le bon déroulement de la traversée et bénissait le navire qui l'effectuait 28 . Des bateaux en terre cuite pouvaient être offerts à cette divinité en étant jetés à la mer, comme en témoignent deux autres modèles, celui de la collection privée de Phr. Nicolaidès, daté du Chypro-Géométrique I ${ }^{29}$, et celui de la collection privée de N. Kirzis, daté du Chypro-Géométrique ou Chypro-Archaïque ${ }^{30}$, sur lequel je reviendrai. Tous deux sont aussi de facture très soignée et ont été découverts au large d'Amathonte.

Ainsi, le roi/prêtre d'Amathonte pourrait, sur le «modèle Kakoulli », être en train d'effectuer une prière sur le bateau, afïn d'invoquer la bienveillance et la protection de la divinité attachée à la navigation. Ou bien simplement bénir le navire, sa possible cargaison et son équipage.

Nous connaissons également l'existence, en Grèce, à l'Âge du Fer, de cultes rendus à une divinité protectrice de la navigation, caractérisés par l'offrande de modèles de bateaux, en bois ou en terre cuite. L'Héraion de Samos, par exemple, a livré plusieurs exemplaires de ces modèles votifs en bois, d'une taille variant de 30 à $50 \mathrm{~cm} 31$. Ces objets ont été mis en relation avec le culte d'Héra en tant que divinité protectrice de la

27. Le dédoublement de la personne du roi sur des images n'est pas unique : c'est le cas, par exemple, sur le vase de Sidon (tout récemment réinterprété par J.-M. Husser) où sont représentés deux fois le roi de Tyr et le dieu Melqart, mais dans deux attitudes ou dimensions cultuelles différentes: J.-M. Husser, "A-t-il existé un bûcher cultuel de Melqart ? ", dans R. Allan, W.G.E. Watson, S. Wiggins (éds), He unfurled his Brow and Laughed... Essays in Honour of Professor Nicolas Wyatt, Münster, sous presse.

28. Dans une étude récente, A. Lemaire présente un fragment de modèle de bateau en "malachite», venant vraisemblablement du commerce et portant une inscription phénicienne: «/... il a b]éni Tyr et sauvé les Sidoniens des nuages épais et n’a pas terrifié le roi Ittobaal fils du roi Hirôm, roi de Tyr ». Cette inscription est datée d'environ 539-533/2 av. J.-C. par l'auteur et elle peut faire référence à l'objet votif lui-même, à savoir la maquette de bateau et la divinité à laquelle elle était offerte en remerciement pour avoir sauvé le roi Ittobaal d'un naufrage lors d'une tempête. Selon l'auteur, "l'absence de parallèle exact ne permet pas de préciser le nom de la divinité qui pourrait avoir été aussi bien le dieu national Milqart, qu'un Baal [...] ou encore une divinité proprement marine ». Pour l'étude détaillée de cette inscription, voir A. Lemaire, "Inscription royale phénicienne sur bateau votif ",
T'Shûrôt LaAvishur 2004, p. 117-129.

29. Karageorghis 1993 , p. 74 , cat. n GK(ii)4, pl. XXXII.

30. V. Karageorghis date ce bateau du Chypro-Géométrique I (Karageorghis 1993, p. 74, cat. $n^{\circ} \mathrm{GK}[i i] 1$, fig. 62); L. Bash propose l'époque archaïque (Basch 1999, p. 60).

31. Voir Kopcke 1967, p. 145-148; Kyrieleis 1980, p. 87-94. 
navigation 32, comme Athéna, Aphaia, Eileithyia ou Aphrodite pouvaient l'être ailleurs. Leur présence est d’autant plus significative que de vrais navires ont également été consacrés dans le sanctuaire à l'époque archaiqque ${ }^{33}$. Des batcaux en terre cuite ont été découverts dans d'autres sanctuaires de la déesse, comme à Pérachora ou à Paestum (Silaris), mais aussi dans des sanctuaires d'autres divinités liées à la navigation, notamment à Inatos en Crète, à Délos, à l'Isthme, à Pithécusses/Ischia. Au sanctuaire d'Aphaia à Égine, ils sont associés à des figurines féminines en terre cuite, porteuses d'une offrande particulière puisqu'il s'agit de bateaux remplis de fleurs 34 .

La déesse Brizo, à Délos, recevail des lemmes de l’île, qui l’imploraient pour obtenir

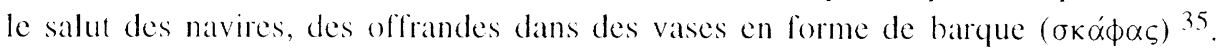
J'aimerais revenir, à cette occasion, sur le modele de la collection Kirzis (Fig. 6). Ce modele, long de $45 \mathrm{~cm}$ et large de $23 \mathrm{~cm}$, se singularise par l'impression de vide qu'il dégage de prime abord. En effet, l’intérieur de la coque est dépourvu de toute trace d'aménagement (saul une emplanture de mât), alors que l'architecture de la coque et le rendu des détails sont très soignés. Ce modèle évoque davantage un grand récipient en forme de navire (un «bateau-réceptacle»?), plutôt qu'un modèle de navire. Une des interprétations possibles serait qu'il ait servi de réeeptacle à des libations et à des offrandes ${ }^{36}$, puis jeté en mer en l'honneur d'une divinité aux connotations maritimes certaines.

Enfin, il faut relever que la coutume de jeter, dans un geste votif, des objets précieux en mer est bien attestée dans l'Antiquité : D. Wachsmuth, dans son étude sur les pratiques religieuses des marins de l'Antiquité, cite, parmi les actes votils non sanglants, le jet à la mer de libations de vin, de miel, d'eau et de nombreux objets précicux 37.

D'autres représentations, mais sur des supports iconographiques complètement différents, vont aussi dans le sens d'une telle interprétation. Sur une coupe en argent à décor gravé conservée à Berlin, qui provient de la collection Cesnola, on observe trois barques de papyrus portant des scrviteurs et des musiciens, accompagnées d'un quatrième bateau, à la proue en protomé d'oiseau, dans lequel trône le maître de la troupe.

32. Voir Kyrieleis 1980 .

33. Voir Kopcke 1967, p. 145 sq4.; Kyrieleis 1980, p. 89-105.

34. Voir U. Sinn (U.), "Der Kult der Aphaia auf Aegina », dans R. Hägg, N. Marinatos, G. Nordquist (éds), Early Greek Cult Practice, Stockholm, 1988, p. 151-152, fig. $2-3$.

35. Basch 1999, p. 62 (Athénée VIII, 335h).

36. Basch 1999, p. 60-61.

37. D. Wachsmuth, Ióknos ó Sxípwv. Untersuchungen zu den antiken Sakralhandlungen bei Serereise, Thèse, 1967. 
P. Monte is. puis J.W. Meger is ont montré qu'il sageissait d'une conpe de la série "chypro-phénicienne plutôt que d'une importation égyptienne. eomme on l'a souvent cru. A. Hermary rapproche la seène figurée sur la coupe de Berlin de celle d'une autre coupe en argent de la collection Cesnolit. conservéc à New York, qui montre sur plusieurs registres une grande fête champêtre. Selon lui t), même si le contenu et le style des représentations sont diférents, il s’agit dans les deux cas d’exuvres réalisées par des artisans phéniciens pour des princes chypriotes, désireux d illustrer le déroulement de fêtes dont ils étaient les protagonistes: « de même que les petits chariots en terre cuite offerts dans les sanctuaires ou déposés dans les tombes rappelaient presque certainement les cérémonies qui avaient lieu sur la terre ferme, de même une bonne partie des bateaux - peut-être tous ceux qui étaient offerts dans les sanctuaires - évoquait probablement des lêtes maritimes célébrées dans les royaumes chypriotes ». A. Hermary interprete, en outre, dans un sens religieux comparable, un document unique en son genre découvert récemment sur le site de Tel Dor, en Israël 41 : il s'agit d'un fragment d'omoplate de bovidé qui porte, sur une face, un décor incisé. Selon lui, il figurerait un bateau arrivant dans un sanctuaire, matérialisé par une colonne et deux personnages qui font un geste d'adoration. Sur l'autre face, une inscription en syllabaire chypriote est une dédicace apparemment plus récente. Il met cet objet en relation avec l'existence possible d'un "pèlerinage» qui conduisait des Chypriotes vers un sanctuaire du Levant. Cette interprétation ne peut toutefois pas être étendue à tous les modèles de bateaux offerts dans les sanctuaires 42 .

Amathonte s'affirme done de plus en plus comme le site chypriote où l'offrande de modèles de bateaux en terre cuite, dans les tombes, les sanctuaires ou jetés à la mer, est la mieux attestée à l'époque archaïque. Le sens de ces objets peut ne pas être univoque, mais revêtir différents aspects selon leur utilisation : liés à la navigation funéraire quand il sont offerts dans une tombe, en relation avec le pouvoir royal quand ils sont découverts sur l'acropole, ou encore, comme ici, bateaux votifs offerts à la mer lors de cérémonies accomplies par le roi/prêtre.

Université Marc-Bloch, Strasbourg

38. P. Montet, Les reliques de l'art syrien dans l'Égypte du Nouvel Empiré, Paris, 1937, p. $151-156$.

39. Dans E. Lipinski (éd.), Studia Phoenicia V, Leuven, 1987, p. 167-180.

40. Hermary 2000 , p. $51-52$.

41. Ibid.

42. L'étude contextuclle complète de tous les modèles de bateaux chypriotes en terre cuite a fait l'objet de mon mémoire de Maîtrise. 


\section{BIBLIOGGRAPHIE}

AUPERT (P.), HILIMANN (M.-Chr.). 1984. Amathonte I, Testimonia I, Auteurs anciens, monnavage, povage'ters. fouilles, origines, géographie, Paris.

BASCH (L.), 1999, "Un modicle de navire chypriote du vi" siècle av. J.-C. trouvé en mer au large d'Amathonte", $B C H \quad 123$, p. 43-64.

GoTTIICHER (A.), 1978, Materiale fiir ein Corpus der Schiffsmodelle im Altertum, Mayence.

Hermary (A.), 20)(0), Amathomte $V$, Les

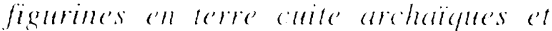
classiques, Le's sculpture's an piorren, Paris.

JONES (D.), 1990, Models of Boats from the Tomb of Tut'Ankhamun, Oxford.

KARAGEORGHIS (V.), 1987, "The Terracottas », dans La nécropole d'Amathonte, tombes 113-367, vol. III, Nicosie, p. 1-52, pl. I-XLI.

Karageorghis (V.), 1993, The Coroplastic Art of Ancient Cyprus II, Late Cypriote II-Cypro-Geometric III, Nicosie.

KARAGLOR(iHIS (V.), 1995, The Coroplastic Art of Ancient Cyprus IV, The CyproArchaic Period, Small Male Figurines, Nicosic.
Karagigorghis (V.), 1996, The Coroplastic Art of Ancient Cyprus VI, The CyproArchaic Pariod Monsters, Animals and Miscellaneous, Nicosie.

Kopcke: (G.), 1967, « Neue Holzfunde von Samos », $A M$ 82, p. 100-148.

KYRIELIAS (H.), 1980, «Archaische Hol\%funde aus Samos" $A M$ 95, p. 87-147.

Monloup (Th.), 1984, Salamine de Chypre XII, Les figurines de terre cuite de tradition archaïque, Paris.

MURRAY (A.S.), SMITH (A.H.), WAITHRS (H.B.), 190(), Excavations in Cyprus, Londres.

PlitT (Th.), 1996, "Religion el royauté à Amathonte de Chypres, Transeuphratene 12, p. 97-109.

PE:TT (Th.), 2003, «Images de la royauté amathousienne: Ic sarcophage d'Amathonte ", dans Y. Perrin, Th. Petit (éds.), Iconographie royale, iconographie impériale, iconographie des élites, Saint-Étienne.

Westerberg (K.), 1983, Cypriote Ships from the Bronze Age to c. 500 B.C., SIMA Pocket-book 22, Göteborg. 

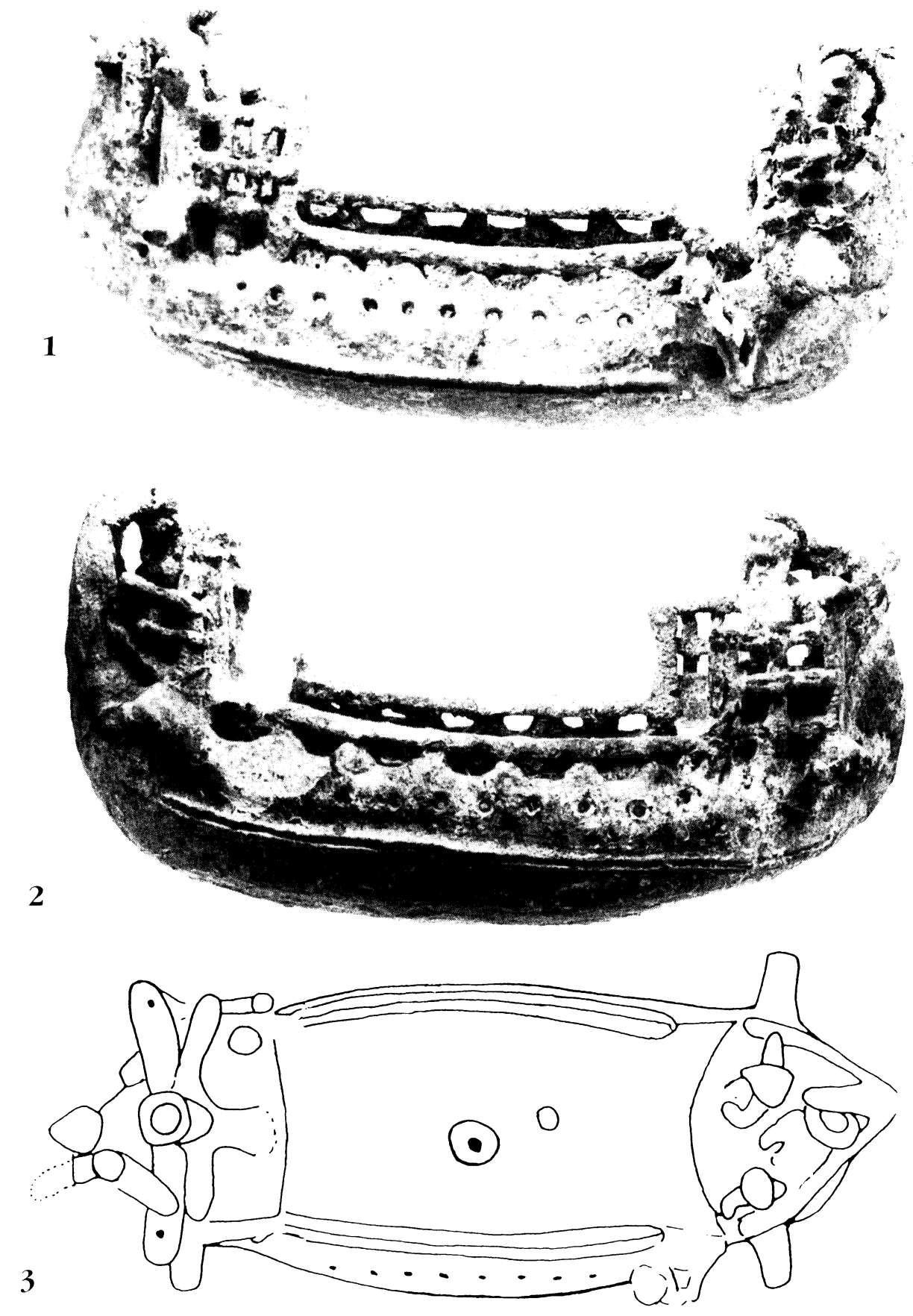

Figures 1-3. « Modèle Kakoulli ».
1. Prolil, bâbord.
2. Profil, tribord.
3. Schéma du modèle.

Musée de la Fondation Piérides, actuellement exposé à Ayia Napa. D’après Basch 1999. 


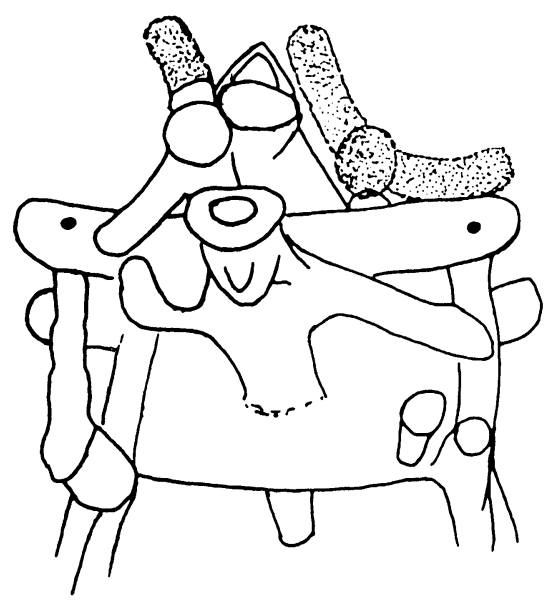

Figure 4. «Modèle Kakoulli »: reconstitution du groupe de trois personnages à l'avant du bateau.

T. : thymiatérion;

en hachuré : les parties manquantes.

D’après Basch 1999.

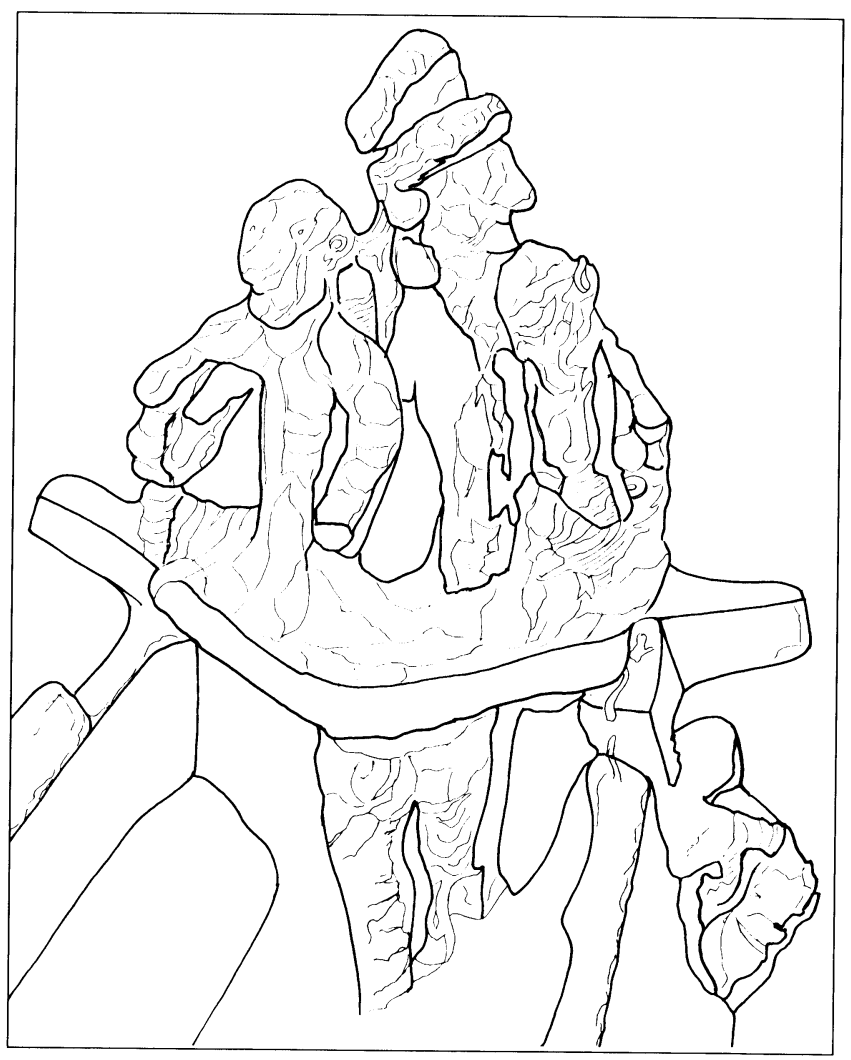

Figure 5. "Modèle Kakoulli », détail : groupe de l'arrière. Dessin M. Rival (Centre Camille Jullian), d'après Basch 1999. 

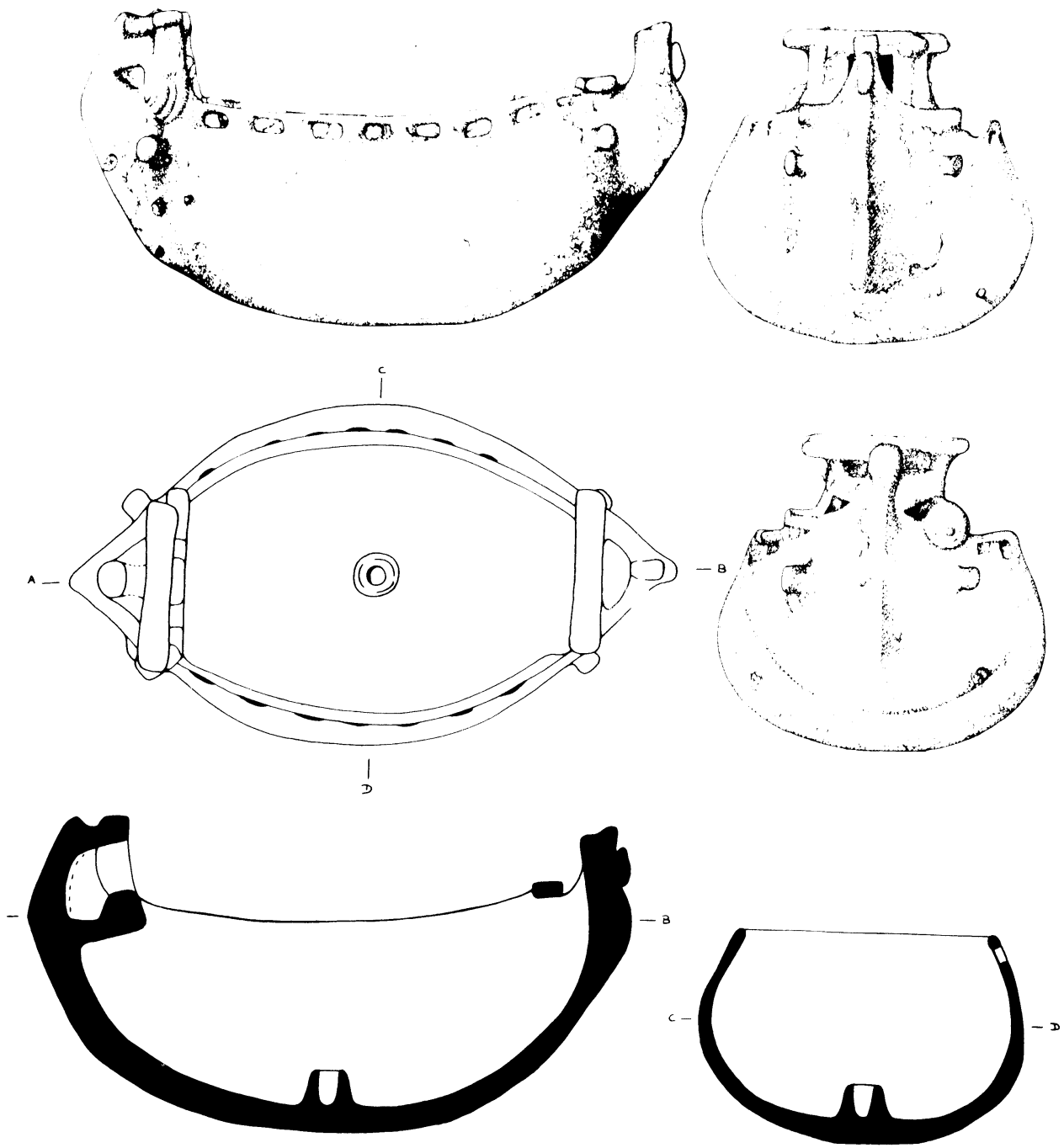

Figure 6. Modele de la collection Kirzis.

D'après Karrageorghis 1993. 